\title{
Manganese spread in Ursa Minor as a proof of sub-classes of type la supernovae
}

\author{
G. Cescutti ${ }^{1, \star}$ and C. Kobayashi ${ }^{2, \star}$ \\ 1 INAF-Osservatorio Astronomico di Trieste, 34131 Trieste, Italy \\ e-mail: cescutti@oats.inaf.it \\ 2 Centre for Astrophysics Research, School of Physics, Astronomy and Mathematics, University of Hertfordshire, College Lane, \\ Hatfield AL10 9AB, UK
}

Received 19 June 2017 / Accepted 24 August 2017

\begin{abstract}
Context. Recently, new sub-classes of Type Ia supernovae (SNe Ia) were discovered, including SNe Iax. The suggested progenitors of $\mathrm{SNe}$ Iax are relatively massive, possibly hybrid $\mathrm{C}+\mathrm{O}+\mathrm{Ne}$ white dwarfs, which can cause white dwarf winds at low metallicities. There is another class that can potentially occur at low or zero metallicities; sub-Chandrasekhar mass explosions in single and/or double degenerate systems of standard $\mathrm{C}+\mathrm{O}$ white dwarfs. These explosions have different nucleosynthesis yields compared to the normal, Chandrasekhar mass explosions.

Aims. We test these SN Ia channels using their characteristic chemical signatures.

Methods. The two sub-classes of SNe Ia are expected to be rarer than normal SNe Ia and do not affect the chemical evolution in the solar neighbourhood; however, because of the shorter delay time and/or weaker metallicity dependence, they could influence the evolution of metal-poor systems. Therefore, we have included both in our stochastic chemical evolution model for the dwarf spheroidal galaxy Ursa Minor.

Results. The model predicts a butterfly-shape spread in $[\mathrm{Mn} / \mathrm{Fe}]$ in the interstellar medium at low metallicity and - at the same time a decrease of $[\alpha / \mathrm{Fe}]$ ratios at lower $[\mathrm{Fe} / \mathrm{H}]$ than in the solar neighbourhood, both of which are consistent with the observed abundances in stars of Ursa Minor.

Conclusions. The surprising agreement between our models and available observations provides a strong indication of the origins of these new sub-classes of SNe Ia. This outcome requires confirmation by future abundance measurements of manganese in stars of other satellite galaxies of our Milky Way. It will be vital for this project to measure not the most extreme metal-poor tail, as more commonly happens, but the opposite; the metal-rich end of dwarf spheroidals.
\end{abstract}

Key words. galaxies: evolution - galaxies: dwarf - stars: abundances - nuclear reactions, nucleosynthesis, abundances supernovae: general

\section{Introduction}

The scenario leading to a supernova Type Ia (SN Ia) explosion is still under debate. The two most common progenitor scenarios are: the single degenerate scenario, in which a white dwarf (WD) with a mass close to the Chandrasekhar $(\mathrm{Ch})$ limit accretes mass from a companion (a red giant or a main sequence star), and the double degenerate scenario in which two WDs merge due to the loss of angular momentum. Both scenarios present pros and cons, but at present neither is able to explain all observational constraints of SNe Ia (e.g. Maoz et al. 2014). Thanks to observational surveys, a large number of SNe Ia are observed as a luminous and (almost) standard candle, and the SN Ia explosions were fundamental to understanding the expansion rate of the Universe and led to the discovery of dark energy (Riess et al. 1998; Schmidt et al. 1998; Perlmutter et al. 1999), after the dismissal by Albert Einstein several decades ago. On the other hand, the surveys have revealed a small variation of the standard candle such as "super-luminous" or "faint", referred to as Type Iax (e.g. Foley et al. 2013).

\footnotetext{
^ BRIDGCE UK Network: www.bridgce.ac.uk/
}

In this paper, we test possible channels in which different scenarios for $\mathrm{SNe}$ Ia are actually real and present themselves in the Universe (or at least in the chemical pollution). Theoretically, SN Ia channels consist of the progenitors and the explosion mechanism, and the former determines the lifetime, while the latter determines the nucleosynthesis yields. Observationally, the majority of SNe Ia seem to be Ch-mass explosions (e.g. Scalzo et al. 2014), such as deflagrations or delayed detonations (Iwamoto et al. 1999). Although both single and double degenerate binary systems have been debated as possible progenitors of SNe Ia, numerical simulations of double-degenerate systems show the explosions to be similar to sub-Ch mass detonations (Pakmor et al. 2012).

The class of SNe Iax, also called 02cx-like, is used for various objects typically $\gtrsim 1$ mag fainter than normal SNe Ia; although SN 2002cx is the prototype (Li et al. 2003), this class includes objects ranging from $M_{V} \sim-19$ to -14 mag at peak (e.g. Miller et al. 2017). There is one detection of the companion star (McCully et al. 2014), and the host galaxies tend to be late type (Foley et al. 2013; White et al. 2015). Although several models have been discussed for particular objects, the preferred model for this class is a single degenerate system of a hybrid, 
$\mathrm{C}+\mathrm{O}+\mathrm{Ne}$ WD (e.g. Meng \& Podsiadlowski 2014). In the stellar evolution of intermediate-mass stars, such hybrid WDs are known, and the outer $\mathrm{O}+\mathrm{Ne}+\mathrm{Mg}$ layer gives a natural reason for the small mass of ejecta with an undisrupted WD after the explosion. The initial mass range of hybrid WDs depends on the metallicity, as well as the mass-loss and nuclear reaction rates. In any case, the rate is expected to be higher for lower metallicities with hybrid WDs. Kobayashi et al. (2015) first included SNe Iax in galactic chemical evolution models, and we use the same mass ranges as adopted in that publication for the primary and secondary stars in this paper. Since the WD mass is already close to the Ch-mass, WD winds can easily occur, and thus a wide range of secondary mass is allowed (Fig. 2 of Kobayashi et al. 2015). Sub-Ch mass C+O WDs $\left(\sim 1 M_{\odot}\right)$ have been invoked to explain some spectral features of SNe Ia (e.g. Shigeyama et al. 1992) and also discussed in relation with the rate of SNe Ia predicted by binary population synthesis (Ruiter et al. 2014). Both single and double degenerate systems are possible. The carbon detonation is triggered by detonation of a thin He layer, and the ejected iron mass is not so different from normal SNe Ia. In the single degenerate systems, the required mass accretion rate is lower than Ch-mass WDs, where there is no WD wind, and the rate does not have a strong dependency on the metallicity (Fig. 1 of Kobayashi et al. 2015). Therefore, these two channels (SNe Iax and sub-Ch SNe Ia) are dominant at low metallicities, which is in contrast to normal SNe Ia (with Ch-mass C+O WDs in this paper) that are inhibited due to the lack of WD winds at $[\mathrm{Fe} / \mathrm{H}]<-1.1$ (Kobayashi et al. 1998; Kobayashi \& Nomoto 2009).

In general, it is not easy to constrain SN Ia scenarios because the chemical outcomes are similar. However, the chemical signature is relatively clear in the case of $\mathrm{Mn}$, which is one of the few chemical elements with just a single stable isotope $\left({ }^{55} \mathrm{Mn}\right)$. Recently, this chemical element has been investigated in Kobayashi et al. (2015), where the presence of three channels (Ch, sub-Ch, and Iax) for the production have been shown to promote a different trend in the chemical evolution for dwarf galaxies, keeping the trend in the case of the solar vicinity model unchanged. A similar analyses was performed, but only for the solar vicinity, in Seitenzahl et al. (2013), and the final results were in favor of the presence of two scenarios for $\mathrm{SNe}$ Ia $(\mathrm{Ch}$ and sub-Ch). Regarding the results for dwarf galaxies, a flat and under solar trend for Sagittarius was obtained by Cescutti et al. (2008), by means of a strong dependence to the metallicity of the $\mathrm{SN}$ Ia yields, maintaining again a good agreement for the solar neighbourhood. The same observational results were obtained for Sculptor, Fornax, Carina and Sextans in North et al. (2012). We note that, however, such a metallicity effect is not expected for the majority of SNe Ia where Mn is synthesised in nuclear statistical equilibrium (Kobayashi et al. 2006, 2015).

The final results are indeed connected to the assumptions regarding the rates of the different channels which are indeed still quite difficult to be constrained from observational data and theoretical one-zone models (e.g. Matteucci 2001). However, in the present work we intend to deal with another possible aspect by using a stochastic model: the spread in the $[\mathrm{Mn} / \mathrm{Fe}]$ ratios. We will show both types of model in Sect. 5 .

Previous theoretical works were able to use the spread observed in the Galactic halo to study the production of chemical elements, in particular neutron capture elements (Tsujimoto et al. 1999; Ishimaru \& Wanajo 1999; Travaglio et al. 2001; Argast et al. 2002, 2004; Karlsson \& Gustafsson 2005; Cescutti 2008; Cescutti \& Chiappini 2010; Cescutti et al. 2013). Indeed stochastic modelling enables us to gain an approximative but quantitative description of the spread produced by the different sources polluting the inter stellar medium (ISM). This is particularly useful for rare sources, and the stochastic modelling of the Galactic halo was able to reproduce, for example, the spread in neutron capture elements - due to the rare $r$-process events (see also Cescutti \& Chiappini 2014; Cescutti et al. 2015) - and the smaller dispersion in the alpha-elements regularly injected into the ISM by SN II explosions at the same time. The impact of rotating massive stars on the chemical evolution of CNO (Cescutti \& Chiappini 2010) and of faint SNe on the evolution of carbon and $\mathrm{Ba}$ (Cescutti et al. 2016) has been also investigated by means of stochastic modelling. We note that, there is the third approach, chemodynamical simulations of galaxies (e.g. Kobayashi \& Nakasato 2011), in which hydrodynamics determine the star formation histories and chemical enrichment takes place inhomogeneously. Since these simulations take a lot of computational time, it is not yet possible to explore all possible sources of chemical enrichment.

In this work we use the stochastic and inhomogeneous chemical evolution models to study the pollution due to SNe Ia, not in the early stage of the formation of the Galactic halo, but in the later stage of a dwarf spheroidal (dSph) galaxy, a satellite of our Galaxy. At present, not many measurements of Mn are present in the literature for stars in $\mathrm{dSph}$ galaxies. We decide to compare our results with Ursa Minor because it is one of the dSph galaxies with the largest number of stars for which Mn is measured (Ural et al. 2015), and therefore we decide to apply our modelling to this case.

A significant effort in this subject has been carried out in North et al. (2012), where the authors measured Mn in stars belonging to four dSph galaxies: Sculptor, Fornax, Carina and Sextans. In this work, they presented an impressive amount of Mn data for Fornax and Sculptor. Indeed, we could have started working on one of these two galaxies; however Sculptor and Fornax are more massive and metal rich (McConnachie 2012) compared to Ursa Minor and it is likely that the signature we are looking for is clearer in fainter and more metal-poor objects. On the other hand, Sextans and Carina have stellar masses and absolute luminosities quite similar to those of Ursa Minor, and we could have developed a model for one of these systems, for which however the amount of data is lower than for Ursa Minor.

\section{SN la scenarios and nucleosynthesis}

In this paper, we consider the same assumptions for subluminous (such as SNe Iax), sub-Ch, and Ch-mass SNe Ia in terms of nucleosynthesis and rates of the events as in Kobayashi et al. (2015). For comparison, we present also results obtained using the SN Ia prescriptions adopted in Cescutti et al. (2008) and Spitoni et al. (2009), which are basically the same prescriptions proposed by the influential Matteucci \& Greggio (1986). We decide to call the former prescriptions "Herts" model, whereas the second is called "Trieste" model, from the location of the groups that have developed each model.

\subsection{Herts model}

One important difference is that in the Kobayashi et al. (2015) work, because of the lack of WD winds (Kobayashi et al. 1998) there is no formation of normal SNe Ia at low metallicity $([\mathrm{Fe} / \mathrm{H}] \leq-1.1)$, where normal SN Ia channel is defined as the single degenerate scenario with near Ch-mass delayed detonations or deflagrations. However, at low metallicity, both sub-Ch $\mathrm{SNe}$ Ia and the new channel called SNe Iax can explode. Both 
SNe Iax and sub-Ch SNe Ia play therefore a role in the production of $\mathrm{Fe}$ and $\mathrm{Mn}$ in $\mathrm{dSphs}$ in this framework, and we have incorporated the table presented in Kobayashi et al. (2015) for metallicity $[\mathrm{Fe} / \mathrm{H}]<-1.1$, given chemical evolution of Ursa Minor does not include stars at higher metallicity.

Therefore, we assume that the SN Iax channel produces $M(\mathrm{Fe})=0.193 M_{\odot}$ and $M(\mathrm{Mn})=3.67 \times 10^{-3} M_{\odot}$ adopting the nucleosynthesis yields of the N5def model in Fink et al. (2014) (see also Kromer et al. 2013). The range of primary star mass is $6.5 M_{\odot}<M_{\text {primary }}<7.5 M_{\odot}$, and the secondary can be either a red giant in the mass range $0.8 M_{\odot}<M_{\text {primary }}<3 M_{\odot}$ or a main sequence star in the mass range $1.6 M_{\odot}<M_{\text {primary }}<6.5 M_{\odot}$, depending on the metallicity. For both configurations the probability that the systems explode as SNe Ia is 0.025 .

For the sub-Ch channel, we assume the nucleosynthesis yields taken from the $1.05 M_{\odot}$ model of Shigeyama et al. (1992): $M(\mathrm{Fe})=0.5643 M_{\odot}$, and $M(\mathrm{Mn})=3.246 \times 10^{-3} M_{\odot}$ at the solar metallicity. These values are really close to the $1.06 M_{\odot}$ model in Sim et al. (2010) and Seitenzahl et al. (2013). In sub-Ch SNe Ia, $\mathrm{Mn}$ is mostly synthesised in incomplete Si-burning, and therefore the Mn yields should depend on metallicity. We include this effect as $M(\mathrm{Mn}) \propto Z^{3}$ as in the calculations of Seitenzahl et al. (2015) and Yamaguchi et al. (2015).

\subsection{Trieste model}

The Trieste model is based on the seminal work of Matteucci \& Greggio (1986), which assumes that the sole normal SNe Ia are present but at all metallicities; the mass of binary systems that can explode as normal $\mathrm{SNe}$ Ia are in the range $3 M_{\odot}<M_{\text {binary }}<16 M_{\odot}$, with the maximum mass of the primary of $8 M_{\odot}$. We assume for this model the W7 yields from Iwamoto et al. (1999), $M(\mathrm{Fe})=0.6 M_{\odot}$ and $M(\mathrm{Mn})=$ $8.87 \times 10^{-3} M_{\odot}$. For Mn yields of SNe Ia we also consider an empirical dependence on the metallicity of the yields as considered in Cescutti et al. (2008):

$Y_{\mathrm{Mn}}(z)=Y_{\mathrm{Mn}}^{\mathrm{Iwamoto}}\left(\frac{z}{z_{\odot}}\right)^{0.65}$

In this model the shortest timescale for the explosion of a SN Ia is the evolutionary timescale of $8 M_{\odot}$ star, which is about $30 \mathrm{Myr}$. We note that this timescale is similar to the timescale of SNe Iax in the Herts model.

We intend to investigate not only the different channels and the different nucleosynthesis yields, but also the fact that the two channels of SNe Ia operating at low metallicity in the Kobayashi framework produce different $[\mathrm{Mn} / \mathrm{Fe}]$ ratios. For this reason, we expect on average an intermediate value, but with the presence of spread in the $[\mathrm{Mn} / \mathrm{Fe}]$. Conversely, the single scenario presented in the Trieste prescriptions will produce either an increase in $[\mathrm{Mn} / \mathrm{Fe}]$ if we consider the standard yields, or a flat trend (see for comparison the results in North et al. 2012) if we consider a dependence on the metallicity of the yields of SNe Ia as considered in Cescutti et al. (2008). In both cases with or without the metallicity dependence, no $[\mathrm{Mn} / \mathrm{Fe}]$ spread in expected.

\subsection{Other model assumptions}

Concerning the nucleosynthesis of the massive stars, we have used in the Trieste model the yields from Woosley \& Weaver (1995) at solar metallicity, but considering the newly produced yields ${ }^{1}$. This has been done to reproduce the previous results obtained in Cescutti et al. (2008), and in particular for Ursa Minor in Ural et al. (2015). On the other hand, in the Herts model, we have used the yields with a metallicity dependency for SN and hypernovae (HN) taken from Kobayashi et al. (2011), considering that half of the stars with $M \geq 20 M_{\odot}$ explode as $\mathrm{HNe}$ as in the model by Kobayashi et al. (2006, 2011, 2015); also in this case, we consider the newly produced material.

Single stars with masses of $M<8 M_{\odot}$, that is, lowintermediate mass stars ending their lives as asymptotic giant branch stars, hardly produce Mn or Fe. Therefore, we do not discuss their yields in detail; however, in this mass range the yields by van den Hoek \& Groenewegen (1997) are considered in the code of both models and they have only a small impact on the evolution of the metallicity.

In the Trieste model, we consider the initial mass function (IMF) by Salpeter (1955), that is, a power law:

$\phi(m) \propto m^{-x}$,

with a single slope $x=1.35^{2}$ for $0.1 M_{\odot} \leq m \leq 50 M_{\odot}$. The Herts model adopts the IMF from Kroupa (2008) which is again a power-law, but with three slopes at different mass ranges: $x=$ 1.3 for $0.5 M_{\odot} \leq m \leq 50 M_{\odot}, x=0.3$ for $0.08 M_{\odot} \leq m \leq 0.5 M_{\odot}$ and $x=-0.7$ for $0.01 M_{\odot} \leq m \leq 0.08 M_{\odot}$.

\section{Chemical evolution models for Ursa Minor}

We start our analysis on the dSph Ursa Minor from the standard chemical evolution model described in Ural et al. (2015), in particular their model $\mathrm{C}$, which takes into account a star formation history based on the observational constraints by Carrera et al. (2002) and infall and winds from the system able to reproduce the metallicity distribution function (MDF) obtained by Kirby et al. (2011, for details, see Sect. 3 Ural et al. 2015). In fact, the $[\mathrm{Mn} / \mathrm{Fe}]$ predicted in the Trieste model with the yields from Cescutti et al. (2008) are exactly the same as the results shown in this paper.

Concerning the chemical evolution for the Herts model, we have assumed a different IMF compared to the Trieste model in order to enable our modelling to reproduce the results obtained in former work (Kobayashi et al. 2015). So, in the Trieste model we have a Salpeter IMF (Salpeter 1955), whereas in Herts model we use the Kroupa (2008). The only other difference between the two models is the SFR efficiency which is increased by a factor of 2.3 in the Herts model to reproduce the observational

1 The newly produced yields for the element $i$ are defined as:

$Y_{\text {new }}(i)=M_{\mathrm{ej}} \times\left(X_{\text {fin }}^{\text {nucl }}(i)-X_{\text {ini }}^{\text {nucl }}(i)\right)$,

where $M_{\mathrm{ej}}$ is the mass ejected by the star, $X_{\text {fin }}^{\text {nucl }}(i)$ the final abundance of the element " $i$ " in the nucleosynthesis computation and $X_{\mathrm{ini}}^{\text {nucl }}(i)$ its original abundance again in the nucleosynthesis computation. The total stellar yields considered in our code for the element " $i$ " are:

$Y_{\mathrm{tot}}(i)=Y_{\text {new }}(i)+M_{\mathrm{ej}} \times X_{\mathrm{ini}}^{\mathrm{CE}}(i)$,

where $X_{\mathrm{ini}}^{\mathrm{CE}}(i)$ is the chemical abundance of the element " $i$ " in the chemical evolution code when the star was born. In this way, the computed ISM is not spuriously polluted by the chemical pattern present in the initial composition of the stellar evolution model.

2 In galactic chemical evolution models, the IMF is usually defined as the mass of stars formed in the mass interval $(m, m+\mathrm{d} m)$, instead of the number of stars formed; for this reason, the Salpeter slope is 1.35 , instead of 2.35 . 
Table 1. Summary of the main differences in the chemical evolution between Herts and Trieste models.

\begin{tabular}{ccccccc}
\hline \hline Model & Fig. & $\begin{array}{c}\text { Normal } \\
\text { SNIa }\end{array}$ & $\begin{array}{c}\text { SubCh } \\
\text { SNIa }\end{array}$ & SNIax & IMF & $\begin{array}{c}\text { SF law (Ural et al. 2015) } \\
v\end{array}$ \\
\hline Trieste & 5 & yes & no & no & Salpeter & $3 \times 10^{-2} \mathrm{Myr}^{-1}$ \\
\hline Herts & 6 & no & yes & yes & Kroupa 08 & $7 \times 10^{-2} \mathrm{Myr}^{-1}$ \\
\hline
\end{tabular}

Notes. In the Herts model the Ch-mass single-degenerate channel is not active for $[\mathrm{Fe} / \mathrm{H}]<-1.1$.

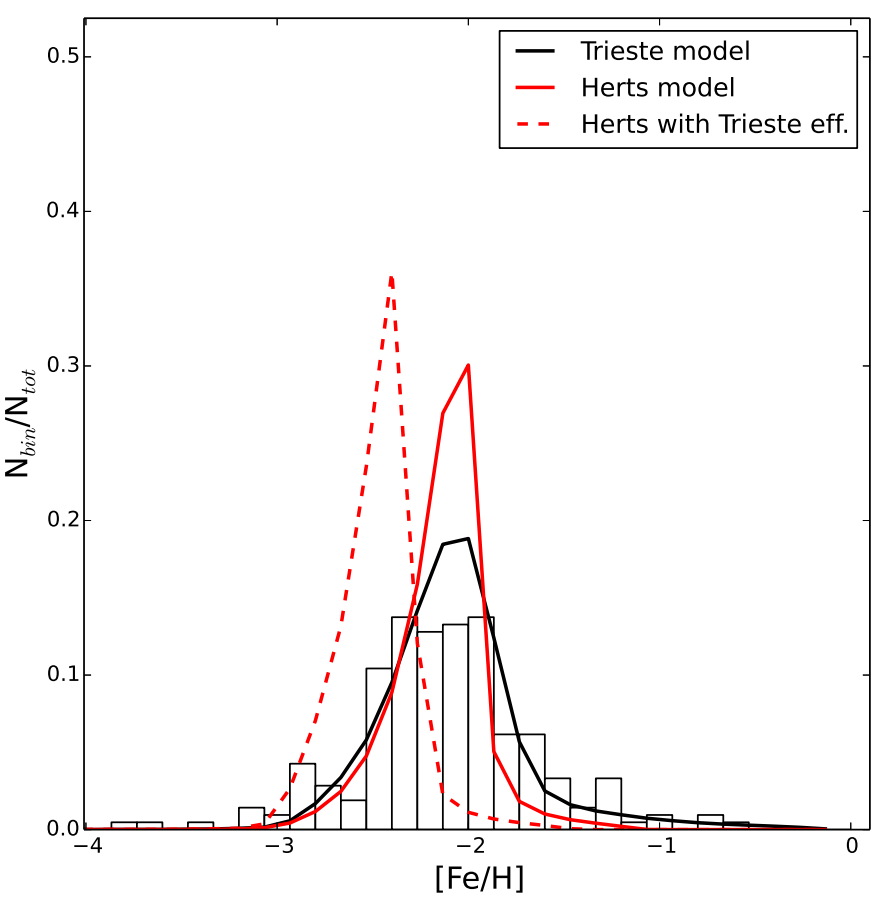

Fig. 1. Metallicity distribution function (MDF) for Ursa Minor; the histogram shows the observational MDF from the data of Kirby et al. (2011). The solid lines are the theoretical MDFs from Trieste (black) and Herts (red) models; the dashed line is the MDF for the Herts model if we keep the star formation efficiency as in the Trieste model.

MDF (cf. Fig. 1). This is because the nucleosynthesis adopted for iron in massive stars is different in the two models; the iron yields in the Trieste model are close to the HN set of yields in the Herts model, but the average production of Fe in Herts model is about a factor of two lower than the Trieste model. For this reason, we have to double the star formation efficiency. With these prescriptions both models are able to reproduce the peak of the MDF and the tail at higher metallicities. Without this correction, and, therefore, with the same efficiency as the Trieste model, the Herts model would not produce a MDF compatible with the observed one, as shown in Fig. 1. A summary of the difference between the Herts and Trieste models is listed in Table 1.

The target of this paper is however, to investigate the possible spread produced by a double channel of Mn production from two different sources. Therefore, we have developed a stochastic chemical evolution model in the same fashion as those implemented in Cescutti (2008), Cescutti et al. (2013) for the Galactic halo, but with the specific star formation history of Ursa Minor, described above. This stochastic modelling enables us to gain an approximative but quantitative description of the spread produced by the different sources polluting the ISM, and is particularly useful for rare sources, such as sub classes of SNe Ia (see also Sect. 1). As we will see, in this model we predict not only a dispersion in the first enrichment by $\mathrm{SNe}$ II, but also later in the

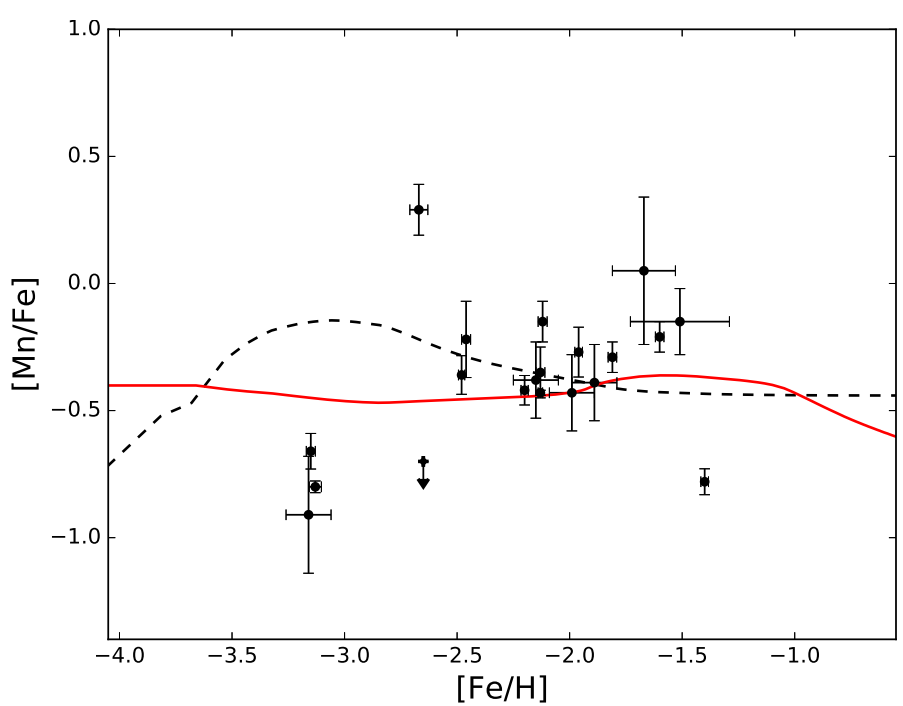

Fig. 2. $[\mathrm{Mn} / \mathrm{Fe}]$ vs. $[\mathrm{Fe} / \mathrm{H}]$; the data in black represent the abundances measured in stars belonging to the dSph Ursa Minor presented in Ural et al. (2015); the red solid line shows the results obtained with the homogeneous chemical evolution by the Herts model, whereas the dashed black line displays the results of the Trieste model.

chemical evolution due to the differential production of Mn by the different SNe Ia channels.

\section{Abundances measured in Ursa Minor stars}

We compare our results with the same set of stars shown in Ural et al. (2015). In this work, the abundances of three stars have been measured and compared to the abundances collected from other authors (Shetrone et al. 2001; Sadakane et al. 2004; Cohen \& Huang 2010; Kirby \& Cohen 2012). We also decided to compare our final results with data coming from another two dSphs similar to Ursa Minor, Sextans and Carina, using the data available in North et al. (2012). The [Mn/Fe] ratios measured in North et al. (2012) are shifted by 0.04 dex to account for the different solar manganese compared to the one adopted in Ural et al. (2015).

\section{Results}

In Figs. 2 and 3, we present our first results, which are the comparison between the results of a homogeneous model of the nucleosynthesis assumed in the Trieste model (as in Ural et al. 2015), and the results of the one assumed in the Herts model (compatible with Kobayashi et al. 2015, but with a specific model for Ursa Minor). These figures do not provide new results, but confirm that the Trieste model reproduces the original model presented in Ural et al. (2015) and the Herts model behaves similarly to the results shown for a generic dSph in Kobayashi et al. (2015). More importantly, the comparison between the two 
G. Cescutti and C. Kobayashi ${ }^{\star}$ : Mn spread in Ursa Minor as proof of sub-classes of type Ia SNe

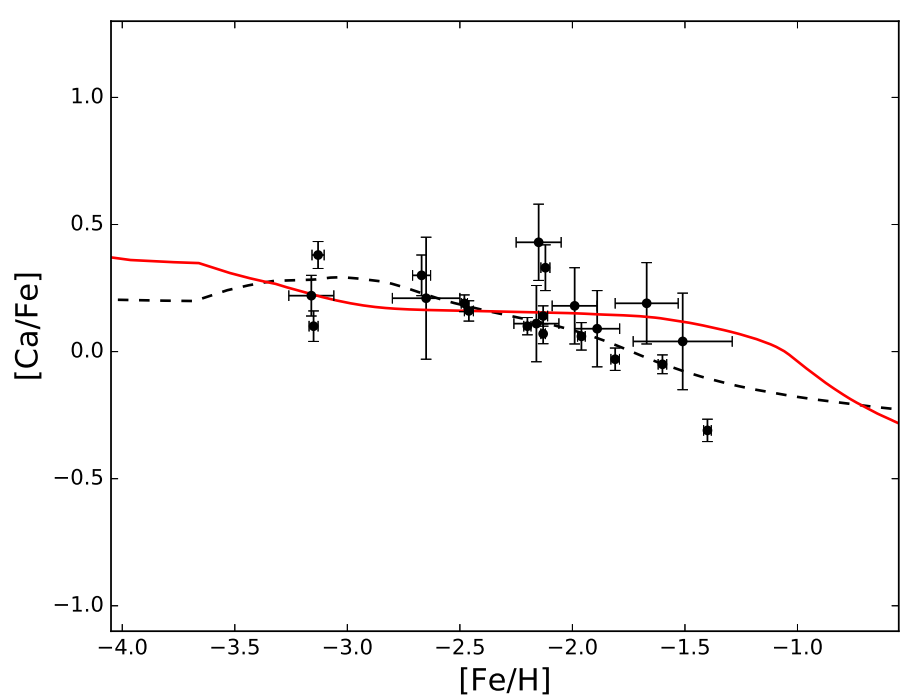

Fig. 3. $[\mathrm{Ca} / \mathrm{Fe}]$ vs. $[\mathrm{Fe} / \mathrm{H}]$; the data in black represent the abundances measured in stars belonging to the dSph Ursa Minor presented in Ural et al. (2015). The red solid line shows the results obtained with the homogeneous chemical evolution by the Herts model, whereas the dashed black line displays the results of the Trieste model.

models shows that in this homogeneous framework both nucleosynthesis processes are quite compatible with the data for this dSph.

The low $[\mathrm{Mn} / \mathrm{Fe}]$, around $-0.5 \mathrm{dex}$ at $[\mathrm{Fe} / \mathrm{H}]>-2.5$, is caused by different phenomena in the two cases. In the Trieste model, it is connected to the metal dependency assumed for the yields of $\mathrm{Mn}$ in SNe Ia. This metal dependency produces a small amount of $\mathrm{Mn}$, if the progenitor has a low metallicity (see Eq. (1)), as is the case for all the progenitor in Ursa Minor. In the other case, the Herts model produces $[\mathrm{Mn} / \mathrm{Fe}]$ that is relatively flat at a level of $[\mathrm{Mn} / \mathrm{Fe}] \sim-0.4$ because it has the two competitive contributions of SNe Ia, producing low $[\mathrm{Mn} / \mathrm{Fe}]$ and high [Mn/Fe] (see Kobayashi et al. 2015, for the models with each contribution).

The behaviour of the two models at extremely low metallicity is not similar, which is driven by the different nucleosynthesis assumed for massive stars. The Trieste model shows relatively large variation, which is due to the dependency on the stellar mass of Mn ejected by the SNe II in the nucleosynthesis adopted for this model (the solar metallicity yields by Woosley \& Weaver 1995). In the Herts model, as discussed in Sect. 2, we assume that, on average, half of the massive stars above $20 M_{\odot}$ explode as SNe II and the rest as HNe. This mix of yields with different energies produces the relatively constant $[\mathrm{Mn} / \mathrm{Fe}]$; we note, however, that similarly to the SN Ia channels, different $[\mathrm{Mn} / \mathrm{Fe}]$ ratios are produced from $\mathrm{SNe}$ II and $\mathrm{HNe}$, but they are averaged in the homogenous chemical evolution model.

The target of these homogenous models is not to analyse the $\mathrm{SNe}$ Ia production of $\mathrm{Mn}$ (and $\mathrm{Fe}$ ), but to show that the SN Ia channels are able to reproduce the trend in $\alpha$ elements. After the Matteucci \& Brocato (1990), it is generally accepted that the delayed SN Ia production is the cause of the knee observed in the $[\alpha / \mathrm{Fe}]$ versus $[\mathrm{Fe} / \mathrm{H}]$ elements in the solar vicinity at around $[\mathrm{Fe} / \mathrm{H}]=-1$ and of a similar knee, but at lower metallicities, for most of the dSph (see Lanfranchi et al. 2008). The same behaviour has been predicted in the Kobayashi et al. (2015). Therefore, in Fig. 3, we show the results of the models for one of the $\alpha$-elements, calcium. Both models have been slightly shifted (Trieste 0.1 dex and Herts -0.15 dex), to have

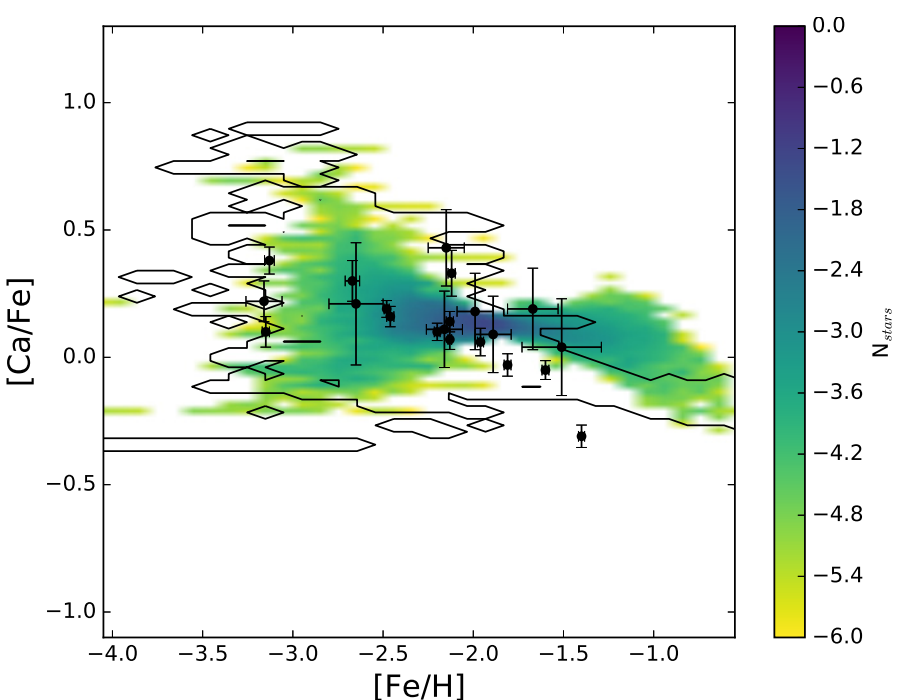

Fig. 4. $[\mathrm{Ca} / \mathrm{Fe}]$ vs. $[\mathrm{Fe} / \mathrm{H}]$; the data are the same as in Fig. 3. Here we show the results with the stochastic modelling. The colour-coded surface density plot presents the density of long-living stars for the Herts model (see colour-bar), whereas the results of the Trieste model are displayed with a black contour.

models pass through $[\mathrm{Ca} / \mathrm{Fe}] \sim 0.25$ at $[\mathrm{Fe} / \mathrm{H}] \sim-2.3$. These small offsets are useful to have a fair comparison between them, assuming $25 \%$ variation in the yields of $\mathrm{Ca}$ can be accommodated in the uncertainties of the stellar model. It could also be the signature of a slightly higher fraction of HN than in the Herts model; in the Trieste model - as mentioned before - this could be due to the explosion modelling that produces too much $\mathrm{Fe}$; indeed dividing the Fe yields by a factor of two has been suggested (Romano et al. 2010).

Both models predict a knee for the $\alpha$-elements in agreement with the abundances measured in the stars of Ursa Minor. In the Trieste model the knee starts early on at $[\mathrm{Fe} / \mathrm{H}] \sim-3.0-$ although part of this trend could be due to the massive stars contribution - and continues with a gentle slope up to $[\mathrm{Fe} / \mathrm{H}] \sim-1$. In the Herts model, it starts only at about $[\mathrm{Fe} / \mathrm{H}] \sim-1.5$ and goes down more quickly. The Trieste model in this respect seems to fit the data slightly better than the Herts one. We recall that in the Herts model at this metallicity there is the contribution of $\mathrm{SNe}$ Iax (producing a low amount of $\mathrm{Fe}$ ) and of sub-Ch $\mathrm{SNe} \mathrm{Ia}$, but there is no formation of normal SNe Ia.

Plots in Figs. 2 and 3 show that both scenarios are able to reproduce the main observed trends within the uncertainties. This confirms that - at least in first approximation - the two models are compatible and it is not possible to distinguish between them by means of a homogenous model.

On the other hand, the use of stochastic modelling could outline a different conclusion, at least for the Mn case. In fact, for the $[\mathrm{Ca} / \mathrm{Fe}]$ versus $[\mathrm{Fe} / \mathrm{H}]$ the stochastic model does not produce significant differences between the two models (see Fig. 4); both Herts and Trieste models can account for most of the data in Ursa Minor, with the possible exception of the star with the lowest $[\mathrm{Ca} / \mathrm{Fe}]$, which appears an outlier in this plot (and possibly for the Mn too).

On the other hand, the situation is different in Figs. 5 and 6, where the $[\mathrm{Mn} / \mathrm{Fe}]$ versus $[\mathrm{Fe} / \mathrm{H}]$ results for the Trieste and Herts models using the stochastic approach are presented.

In the Trieste model (Fig. 5), the strong dependence of $[\mathrm{Mn} / \mathrm{Fe}]$ on the stellar mass in massive stars produces a large spread for $[\mathrm{Fe} / \mathrm{H}]<-2$. On the other side, starting from 

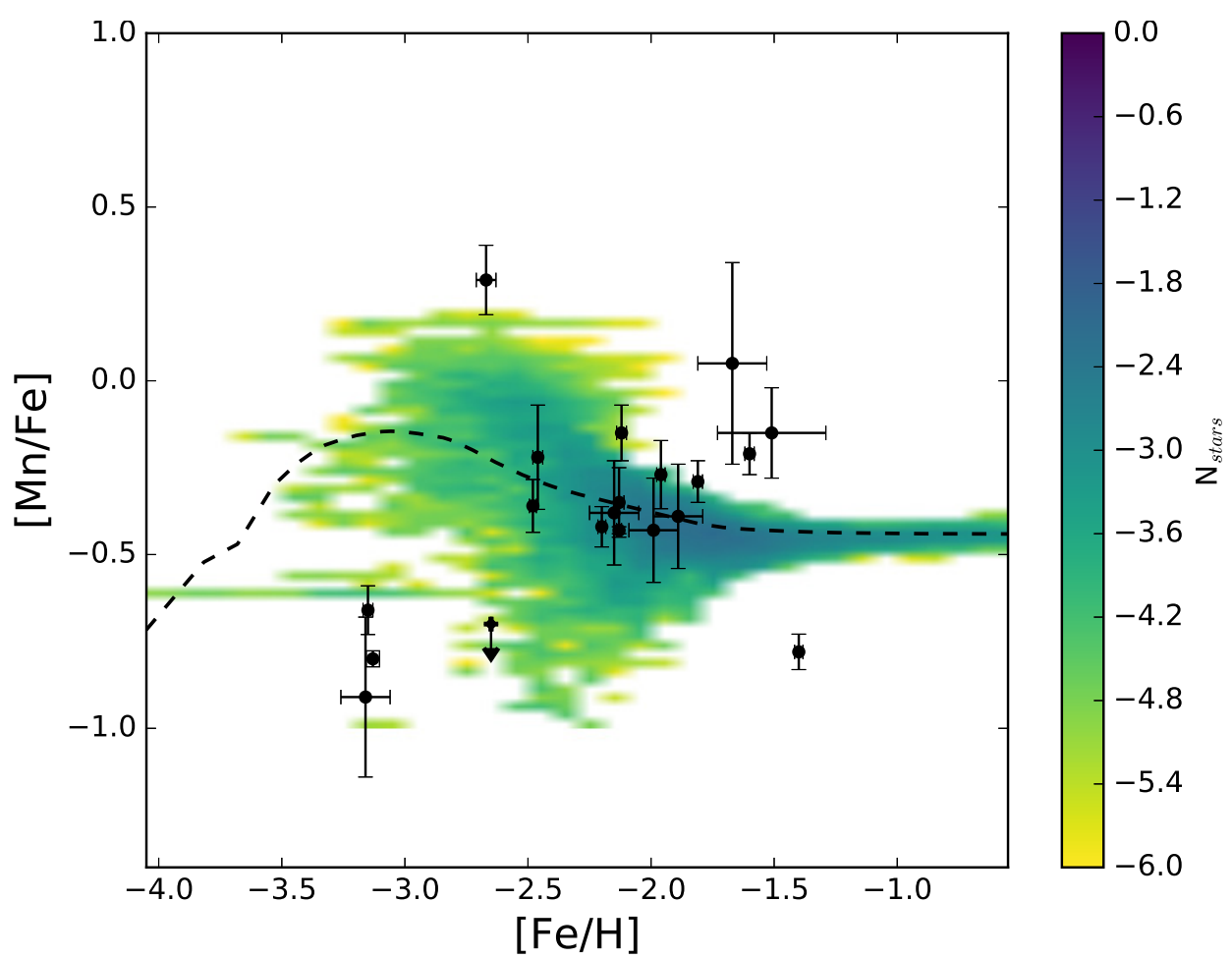

Fig. 5. $[\mathrm{Mn} / \mathrm{Fe}]$ vs. $[\mathrm{Fe} / \mathrm{H}]$; the data in black represent the abundances measured in stars belonging to the dSph Ursa Minor presented in Ural et al. (2015). The colour-coded surface density plot presents the density of long-living stars for the Trieste model.
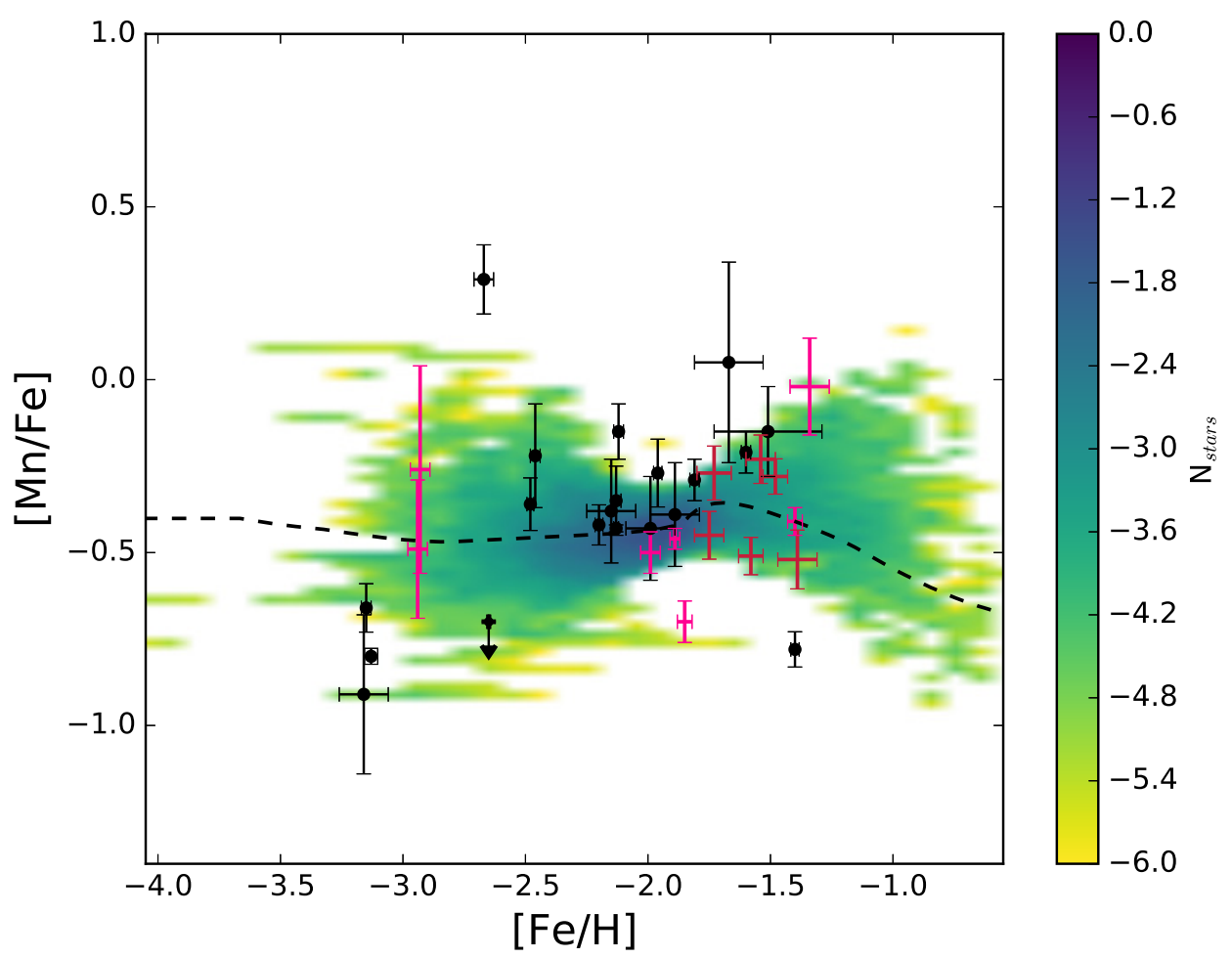

Fig. 6. $[\mathrm{Mn} / \mathrm{Fe}]$ vs. $[\mathrm{Fe} / \mathrm{H}]$; the data in black represent the abundances measured in stars belonging to the dSph Ursa Minor presented in Ural et al. (2015); in red we present the data for the dSph Carina from North et al. (2012) and in magenta those for the dSph Sextans again from from North et al. (2012) and Tafelmeyer et al. (2010). The colour-coded surface density plot presents the density of long-living stars for the Herts model.

$[\mathrm{Fe} / \mathrm{H}] \sim-2$ the spread is relatively reduced, and this is due to the almost fixed enrichment in $[\mathrm{Mn} / \mathrm{Fe}]$ produced by the SNe Ia. The agreement with most of the abundances of $[\mathrm{Mn} / \mathrm{Fe}]$ measured in Ursa Minor - around $[\mathrm{Mn} / \mathrm{Fe}] \sim-0.4$ and $[\mathrm{Fe} / \mathrm{H}] \sim-2$ - is relatively good. The spread of the Trieste model at extremely low metallicity can also explain the extremely high value of one star with $[\mathrm{Mn} / \mathrm{Fe}]>0$ at $[\mathrm{Fe} / \mathrm{H}] \sim-2.7$ and also but at the edge of the probability distribution - the three stars with $[\mathrm{Mn} / \mathrm{Fe}]<-0.5$ (and the star with just an upper limit). At $[\mathrm{Fe} / \mathrm{H}]>-2$, when the SNe Ia start to play an important role, the spread for the Trieste model in the $[\mathrm{Mn} / \mathrm{Fe}]$ versus $[\mathrm{Fe} / \mathrm{H}]$ space is decreased, due to the approximately constant enrichment of $\mathrm{Mn}$ and $\mathrm{Fe}$ from the $\mathrm{SNe} \mathrm{Ia}$. In the region $[\mathrm{Fe} / \mathrm{H}]>-1.8$, the model does not agree with any of the abundances measured in the four stars.

The Herts model with its stochastic results shown in Fig. 6 displays a butterfly shape distribution with remarkable differences compared to the Trieste model.

Again the bulk of the data for Ursa Minor at $[\mathrm{Fe} / \mathrm{H}] \sim$ -2 are within the prediction of the model; moreover at lower metallicities most of the measured stars are in good agreement with the model, and in this case the four stars at $[\mathrm{Fe} / \mathrm{H}]<-2.5$ 
and $[\mathrm{Mn} / \mathrm{Fe}]<-0.5$ are more clearly within the limits of the probability predicted by the model. In this region there is, however, a clear outlier: the star with a $[\mathrm{Mn} / \mathrm{Fe}]>0$. For such a small sample, excluding one star may not be ideal. We note that, however, this star is the only star with a $[\mathrm{Mn} / \mathrm{Fe}]>0$ at $[\mathrm{Fe} / \mathrm{H}]<-2.5$ in Ursa Minor, also in other dSphs (North et al. 2012), and possibly the Milky Way; therefore, this measurement may suffer from some issues. Nevertheless, we consider this star as a special case and conclude that this model is successful in this range of metallicity. The most striking difference is on the high-metallicity tail. In this region, at $[\mathrm{Fe} / \mathrm{H}]>-2$, the model produces again a spread (the right wing of the butterfly). This is due to the onset of the two SN Ia channels (SNe Iax and sub-Ch $\mathrm{SNe}$ Ia) that start to enrich the ISM; we recall that at this metallicity, in the Herts model there is no formation of normal SNe Ia. Producing different $[\mathrm{Mn} / \mathrm{Fe}]$ ratios, they create a spread in the model results: regions polluted by $\mathrm{SNe}$ Iax are extended toward solar $[\mathrm{Mn} / \mathrm{Fe}]$ ratios, the contrary for sub-Ch $\mathrm{SNe}$ Ia that give low $[\mathrm{Mn} / \mathrm{Fe}]$.

Comparing the data we have for Ursa Minor, it clearly appears that the Herts model is the one that more closely approximates what is displayed by the stars of this galaxy. In fact, for $[\mathrm{Fe} / \mathrm{H}]>-1.8$, three stars have a $[\mathrm{Mn} / \mathrm{Fe}]>-0.5$ and one instead has a lower value, presenting therefore a spread. At this stage, the amount of data is not yet great enough to ensure a statistically sound prediction, and a future observational campaign to measure more spectra of stars in Ursa Minor is encouraged. Moreover, we underline that it will be vital for this project to measure not the most extreme metal-poor tail, as commonly happens, but the opposite, the metal rich end, in order to disentangle this problem.

Again, we note that the average effect of the two channels considered in the Herts model are compatible with the average output of the Trieste model, and therefore only in faint satellites such as Ursa Minor can this difference be clearly found. In fact, the expectation is to see this spread being less extreme for galaxies more massive and with higher average metallicities. The reason for this expectation comes from two motivations: first, the impact of the stochasticity is less important at higher metallicities, mixing up the enrichment of the SNe Ia with more preexisting $\mathrm{Mn}$ and Fe coming from massive stars; this point can be proven to be wrong depending on the way the metals are removed by winds (or other mechanism that have acted to deplete gas from these objects) compared to the timescale of the SN Ia explosions. The second point is that nucleosynthesis calculations predict that the amount of $\mathrm{Mn}$ produced by the sub-Ch channel is dependent on the metallicity and therefore at higher metallicity the difference between the two channels is weaker and spread could be reduced. So, the possible smaller spread in larger $\mathrm{dSph}$ galaxies could still be investigated as well as in the Galactic halo; it is necessary to develop specific models to study this cases.

As mentioned in the Introduction, not many measurements of $\mathrm{Mn}$ are present in the literature for stars in dSph galaxies. In this respect a significant amount of work has been carried out by North et al. (2012), where the measurement of four dSph galaxies are presented: Sculptor, Fornax, Carina and Sextans. It appears that also for these galaxies the spread is present, although specific chemical evolution models are required to check our theory against these data. This is particularly true in the case of Sculptor and Fornax that are more massive (McConnachie 2012); on the other hand, Sextans and Carina have stellar masses and absolute luminosity quite similar to those of Ursa Minor.
For this reason, in Fig. 6 we include also the stars measured for these two dSph galaxies in North et al. (2012) and Tafelmeyer et al. (2010); 13 more data points in total. Moreover, eight of these data points sit at $[\mathrm{Fe} / \mathrm{H}]>-1.8$, significantly increasing the only four data points available for Ursa Minor in this metallicity range, where the new Herts model predicts a spread in $[\mathrm{Mn} / \mathrm{Fe}]$. Although the data belong to two different dSphs, it is still encouraging to see that the model is in excellent agreement with them; in fact the data for Sextans, for example, show a remarkable spread of more than 0.5 dex.

Therefore, we encourage more investigations to establish the presence of this spread in the $[\mathrm{Mn} / \mathrm{Fe}]$ versus $[\mathrm{Fe} / \mathrm{H}]$ space in other satellites of the Milky Way, in particular in the faint classical dSphs such as Ursa Minor, Sextans, Carina and Draco. On the other hand, we underline that we cannot use the faintest satellite galaxies (or ultra faint galaxies) to confirm this scenario. In fact, it is not clear whether all of these objects evolve enough in time to see the impact in their stellar population of the chemical enrichment by SNe Ia.

\section{Conclusions}

We present new results for the chemical evolution of the $[\mathrm{Mn} / \mathrm{Fe}]$ in a relatively faint $\mathrm{dSph}$ galaxy, Ursa Minor, with two different prescriptions for SNe Ia. These two prescriptions, that we call Herts and Trieste, present the following differences: in the Trieste model, we allow only a single channel of SNe Ia to explode, the single degenerate with a deflagration; in the Herts model we have two different channels, one is a sub-Ch channel, with a double detonation, the other is a special case of a single degenerate, originating from a relatively massive primary star, producing a relatively weak deflagration (SN Iax channel). These two channels produce, on average, almost the same amount of $\mathrm{Mn}$ as the the single channel in the Trieste model. We show that in the framework of a homogenous chemical evolution model, both Herts and Trieste prescriptions are compatible with the data available for $\mathrm{Mn}$ in this $\mathrm{dSph}$. On the other hand, in the stochastic framework, the results are different and the data favour the Herts model, and therefore, the presence of two channels for SNe Ia at low metallicity, in addition to normal SNe Ia at higher metallicities. This conclusion is supported also by the chemical evolution results obtained for the solar vicinity case in Seitenzahl et al. (2013). Including the data for another two faint dSphs with similar characteristics to Ursa Minor, Sextan and Carina, confirms the necessity of these two channels to explain the data, although specific chemical evolution models for these galaxies would be best suited to check this. We plan to extend our studies also to these galaxies as well as more massive dSphs like Sculptor to confirm this result. Clearly, these results need to be confirmed by future abundance measurements of manganese in stars of other satellite galaxies of our Milky Way. It will be vital for this project to measure not the most extreme metal-poor tail, as more commonly happens, but the opposite; the metal-rich end of dSph galaxies.

Acknowledgements. This work was supported by the Science and Technology Facilities Council ST/M000958/1 for the BRIDGCE consortium grant. G.C. acknowledges financial support from the European Union Horizon 2020 research and innovation programme under the Marie Skłodowska-Curie grant agreement No. 664931.

\section{References}

Argast, D., Samland, M., Thielemann, F.-K., \& Gerhard, O. E. 2002, A\&A, 388, 842 
Argast, D., Samland, M., Thielemann, F.-K., \& Qian, Y.-Z. 2004, A\&A, 416, 997

Carrera, R., Aparicio, A., Martínez-Delgado, D., \& Alonso-García, J. 2002, AJ, 123,3199

Cescutti, G. 2008, A\&A, 481, 691

Cescutti, G., \& Chiappini, C. 2010, A\&A, 515, A102

Cescutti, G., \& Chiappini, C. 2014, A\&A, 565, A51

Cescutti, G., Matteucci, F., Lanfranchi, G. A., \& McWilliam, A. 2008, A\&A 491, 401

Cescutti, G., Chiappini, C., Hirschi, R., Meynet, G., \& Frischknecht, U. 2013 A\&A, 553, A51

Cescutti, G., Romano, D., Matteucci, F., Chiappini, C., \& Hirschi, R. 2015 A\&A, 577, A139

Cescutti, G., Valentini, M., François, P., et al. 2016, A\&A, 595, A91

Cohen, J. G., \& Huang, W. 2010, ApJ, 719, 931

Fink, M., Kromer, M., Seitenzahl, I. R., et al. 2014, MNRAS, 438, 1762

Foley, R. J., Challis, P. J., Chornock, R., et al. 2013, ApJ, 767, 57

Ishimaru, Y., \& Wanajo, S. 1999, ApJ, 511, L33

Iwamoto, K., Brachwitz, F., Nomoto, K., et al. 1999, ApJS, 125, 439

Karlsson, T., \& Gustafsson, B. 2005, A\&A, 436, 879

Kirby, E. N., \& Cohen, J. G. 2012, AJ, 144, 168

Kirby, E. N., Lanfranchi, G. A., Simon, J. D., Cohen, J. G., \& Guhathakurta, P 2011, ApJ, 727, 78

Kobayashi, C., \& Nomoto, K. 2009, ApJ, 707, 1466

Kobayashi, C., \& Nakasato, N. 2011, ApJ, 729, 16

Kobayashi, C., Tsujimoto, T., Nomoto, K., Hachisu, I., \& Kato, M. 1998, ApJ, 503, L155

Kobayashi, C., Umeda, H., Nomoto, K., Tominaga, N., \& Ohkubo, T. 2006, ApJ, 653,1145

Kobayashi, C., Karakas, A. I., \& Umeda, H. 2011, MNRAS, 414, 3231

Kobayashi, C., Nomoto, K., \& Hachisu, I. 2015, ApJ, 804, L24

Kromer, M., Fink, M., Stanishev, V., et al. 2013, MNRAS, 429, 2287

Kroupa, P. 2008, in Pathways Through an Eclectic Universe, eds. J. H. Knapen, T. J. Mahoney, \& A. Vazdekis, ASP Conf. Ser., 390, 3

Lanfranchi, G. A., Matteucci, F., \& Cescutti, G. 2008, A\&A, 481, 635

Li, W., Filippenko, A. V., Chornock, R., et al. 2003, PASP, 115, 453

Maoz, D., Mannucci, F., \& Nelemans, G. 2014, ARA\&A, 52, 107
Matteucci, F. 2001, Nature, 414, 253

Matteucci, F., \& Brocato, E. 1990, ApJ, 365, 539

Matteucci, F., \& Greggio, L. 1986, A\&A, 154, 279

McConnachie, A. W. 2012, AJ, 144, 4

McCully, C., Jha, S. W., Foley, R. J., et al. 2014, Nature, 512, 54

Meng, X., \& Podsiadlowski, P. 2014, ApJ, 789, L45

Miller, A. A., Kasliwal, M. M., Cao, Y., et al. 2017, ApJ, submitted [arXiv: 1703.07449]

North, P., Cescutti, G., Jablonka, P., et al. 2012, A\&A, 541, A45

Pakmor, R., Kromer, M., Taubenberger, S., et al. 2012, ApJ, 747, L10

Perlmutter, S., Aldering, G., Goldhaber, G., et al. 1999, ApJ, 517, 565

Riess, A. G., Filippenko, A. V., Challis, P., et al. 1998, AJ, 116, 1009

Romano, D., Karakas, A. I., Tosi, M., \& Matteucci, F. 2010, A\&A, 522, A32

Ruiter, A. J., Belczynski, K., Sim, S. A., Seitenzahl, I. R., \& Kwiatkowski, D. 2014, MNRAS, 440, L101

Sadakane, K., Arimoto, N., Ikuta, C., et al. 2004, PASJ, 56, 1041

Salpeter, E. E. 1955, ApJ, 121, 161

Scalzo, R. A., Ruiter, A. J., \& Sim, S. A. 2014, MNRAS, 445, 2535

Schmidt, B. P., Suntzeff, N. B., Phillips, M. M., et al. 1998, ApJ, 507, 46

Seitenzahl, I. R., Cescutti, G., Röpke, F. K., Ruiter, A. J., \& Pakmor, R. 2013, A\&A, 559, L5

Seitenzahl, I. R., Summa, A., Krauß, F., et al. 2015, MNRAS, 447, 1484

Shetrone, M. D., Côté, P., \& Sargent, W. L. W. 2001, ApJ, 548, 592

Shigeyama, T., Nomoto, K., Yamaoka, H., \& Thielemann, F.-K. 1992, ApJ, 386, L13

Sim, S. A., Röpke, F. K., Hillebrandt, W., et al. 2010, ApJ, 714, L52

Spitoni, E., Matteucci, F., Recchi, S., Cescutti, G., \& Pipino, A. 2009, A\&A, 504, 87

Tafelmeyer, M., Jablonka, P., Hill, V., et al. 2010, A\&A, 524, A58

Travaglio, C., Galli, D., \& Burkert, A. 2001, ApJ, 547, 217

Tsujimoto, T., Shigeyama, T., \& Yoshii, Y. 1999, ApJ, 519, L63

Ural, U., Cescutti, G., Koch, A., et al. 2015, MNRAS, 449, 761

van den Hoek, L. B., \& Groenewegen, M. A. T. 1997, A\&AS, 123, 305

White, C. J., Kasliwal, M. M., Nugent, P. E., et al. 2015, ApJ, 799, 52

Woosley, S. E., \& Weaver, T. A. 1995, ApJS, 101, 181

Yamaguchi, H., Badenes, C., Foster, A. R., et al. 2015, ApJ, 801, L31 